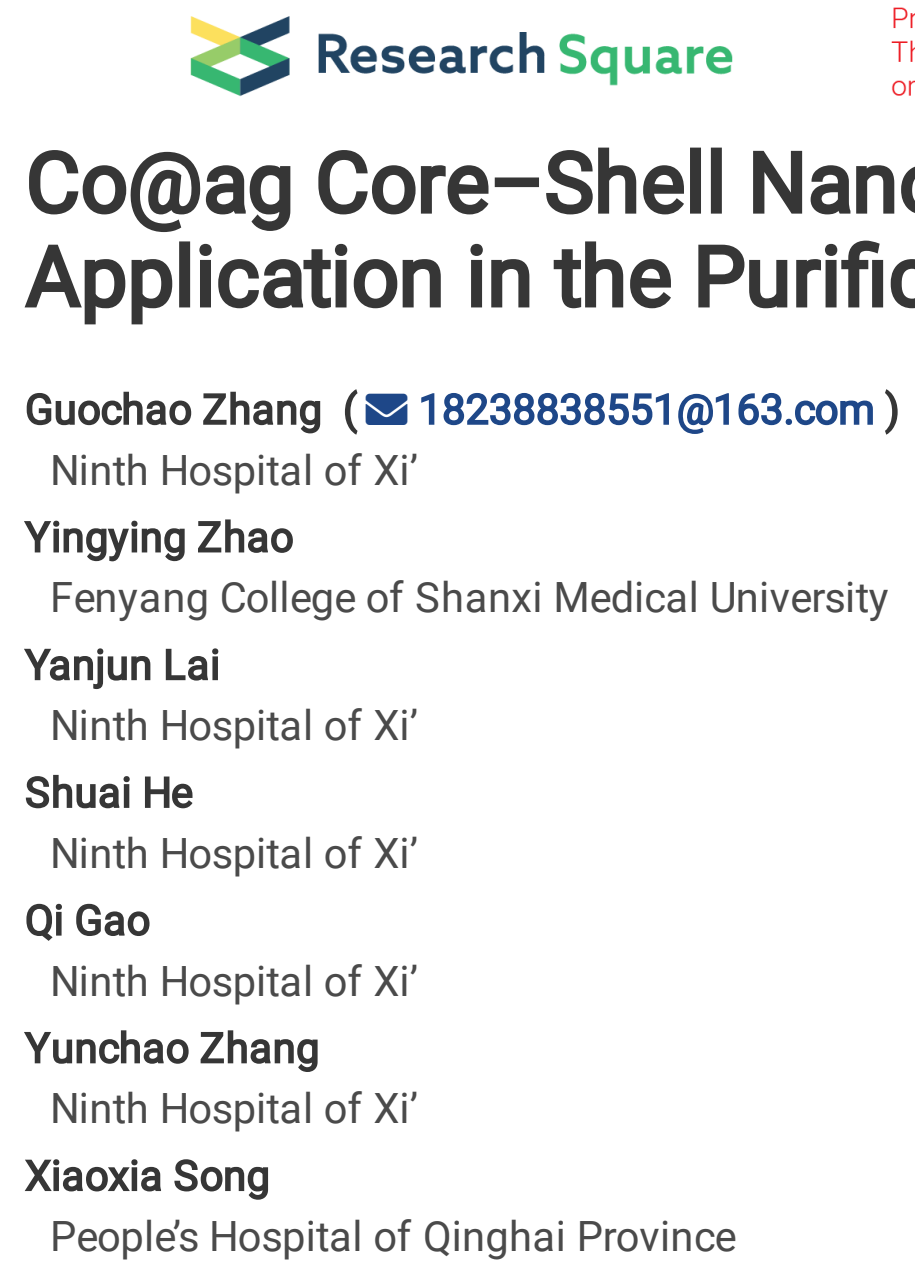

\author{
Guochao Zhang ( $\sim 18238838551 @ 163 . c o m)$ \\ Ninth Hospital of Xi' \\ Yingying Zhao \\ Fenyang College of Shanxi Medical University \\ Ninth Hospital of Xi' \\ Shuai He \\ Ninth Hospital of Xi' \\ Qi Gao \\ Ninth Hospital of Xi' \\ Yunchao Zhang \\ Ninth Hospital of Xi' \\ Xiaoxia Song \\ People's Hospital of Qinghai Province
}

\section{Yanjun Lai}

\title{
Co@ag Core-Shell Nanomaterials: Preparation and Application in the Purification of Yellow River Water
}

\section{Research Article}

Keywords: Co@Ag core-shell nanomaterials, Antibacterial, Yellow River

Posted Date: July 8th, 2021

DOI: https://doi.org/10.21203/rs.3.rs-685814/v1

License: (c) (i) This work is licensed under a Creative Commons Attribution 4.0 International License.

Read Full License 


\section{Abstract}

In this study, we prepared a new type of recyclable Co@Ag core-shell nanomaterials with a very simple method named displacement reaction. Co@Ag core-shell nanomaterials were prepared in $\mathrm{AgNO}_{3}$ solution with cobalt nanomaterials as the core, while the surface was coated with silver nanomaterials. Co@Ag core-shell nanomaterials were characterized by transmission electron microscopy (TEM), X-ray photoelectron spectroscopy (XPS), X-ray diffraction (XRD) and oscillating sample magnetometer (VSM).We found that the diameter of the Co@Ag core-shell nanomaterials was about 100nm, which was exhibited in form of elemental elements andobvious magnetic properties.

In order to evaluate the bacteriostatic effect of Co@Ag core-shell nanomaterials, we used qualitative and quantitative methods to assess the bacteriostatic effect of these materials on standard strains of Pseudomonas aeruginosa (ATCC27853, Gram - negative bacterium), Staphylococcus aureus (ATCC 25923, Gram-positive bacterium), Escherichia coli (ATCC 25922, Gram-negative bacterium) and Candida albicans (ATTC 90029, yeast) in Yellow River water in vitro. The results show that Co@Ag core-shell nanomaterials have a significant antibacterial effect. In addition, Co@Ag core-shell nanomaterials can be recycled through an external magnetic field to reduce environmental pollution. Interestingly, the reclaimed Co@Ag core-shell nanomaterials can still have antibacterial activity and can be reused. These results indicate that Co@Ag core-shell nanomaterials may have potential application as disinfectants for water.

\section{Introduction}

The infection and spread of pathogenic bacteria have always been a threat to public health. Although the threat of pathogenic bacteria has been controlled in developed countries, drug-resistant bacteria, new pathogenic bacteria, and genetically mutated superbugs have emerged with the abuse of antibiotics ${ }^{1}$. In recent years, nanoscaled antibacterial materials are expected to become significant antimicrobial elements due to their novel physical and chemical potential, more importantly, its broad-spectrum antimicrobial activity. Currently, nanoscaled antibacterial materials include $\mathrm{TiO}_{2}, \mathrm{ZnO}, \mathrm{CuO}, \mathrm{Ca}(\mathrm{HO})_{2}, \mathrm{MgO}$, chitosan, silver, and others ${ }^{2-8}$. Among them, nanocrystalline silver and its compounds can efficiently inhibit or even kill pathogenic bacteria, viruses and other microorganisms, which has the advantage of lower toxicity and side effects and plays an important role in local bacteriostatic therapy. Clinically, nanocrystalline silver-loaded medical products can kill bacteria on wounds to minimize infections, thus promoting the healing of chronic wounds ${ }^{9}$. Surgical sutures coated with silver-loaded medical products have a stronger ability to inhibit bacterial adhesion compared with uncoated surgical sutures ${ }^{8}$. In addition, nanocrystalline silver-loaded antibacterial materials are also applied in ureteral catheters, fiber fabrics, antibacterial plastics, and other materials ${ }^{10-12}$. Nanocrystalline silver as the antimicrobial agent is marked by broad-spectrum antibacterial activity, which can kill many kinds of pathogenic microorganisms, and will not lead to microbial resistance ${ }^{13}$. 
In terms of sewage treatment, $\mathrm{TiO}_{2}$ has been used in wastewater treatment systems for a long time; however, excessive accumulation of silver and silver-containing compounds in the body will bring about serious silver poisoning thereby extra time and cost must be paid to deal with these residual chemical materials. Therefore, it is limited in its application prospect with few studies existing on the application of silver-containing compounds in sewage treatment. To solve this problem, people propose that the nanocrystalline silver can be coated on the surface of magnetic materials, and then the silver can be recycled by external magnetic force, easily and efficiently after wastewater treatment. In this course, people can not only efficiently treat wastewater, but also can reclaim the silver-containing compounds to avoid environmental pollution and save costs ${ }^{14-16}$. Cobalt is a kind of amphoteric metal which is identified with good magnetism, similar to iron and nickel in hardness, has suitable tensile strength, machinability, thermodynamic properties and electrochemical behavior. Cobalt especially has obvious advantages in magnetism, being one of the few metals that can remain magnetic after being magnetized once $^{17}$.

In this study, cobalt nanomaterials were used as carriers to prepare silver-loaded Co@Ag core-shell nanomaterials in aqueous solution, as shown in Figure 1-a. These materials have both the antibacterial bioactivities of silver and the magnetic properties of cobalt to ensure that the nanomaterials can be recycled through the external magnetic field. Furthermore, the Co@Ag core-shell nanomaterials bring out significant antibacterial effects on the standard strain, with the growth of bacteria in water of the Yellow River also being inhibited Figure 1b,c. The results show that the magnetic Co@Ag core-shell nanomaterials have a significant antibacterial effect, and promisingly can be used as a new type of recyclable antibacterial agent.

\section{Materials And Methods}

\section{Main reagents}

Reagents were obtained as follows: Cobalt nanomaterials (particle size 100nm) (Shanghai Yunfu Technology Co., Ltd), anhydrous ethanol $\left(\mathrm{C}_{2} \mathrm{H}_{5} \mathrm{OH}\right)$ (Tianjin Baishi Chemical Co., Ltd), silver nitrate $\left(\mathrm{AgNO}_{3}\right)$ (Sinopharm Chemical Reagent Co., Ltd), Mueller-Hinton (M-H) AGAR culture medium (Wenzhou Kangtai Biotechnology Co., Ltd), LB liquid culture medium (dry powder (Beijing Solarbio Science and Technology Co., Ltd). Yellow River water was obtained from the Lanzhou section of the Yellow River.

\section{Standard strains}

Pseudomonas aeruginosa (ATCC27853, Gram-negative bacteria), Escherichia coli (ATCC25922, Gramnegative bacteria), Staphylococcus aureus (ATCC25923, Gram-positive bacteria) and Candida albicans (ATTC90029, yeast) were stored in the Microbiology Laboratory of Xi 'an Ninth Hospital.

\section{Treatment of the Yellow River water}


Firstly, large debris were removed from the Yellow River water through 200-mesh sieve. Then, the Yellow River water stood for precipitation to obtain the supernatant under sterile conditions. A volume of $100 \mu l$ Yellow River water supernatant was absorbed and evenly applied on LB solid medium, which was removed to be cultured at $37^{\circ} \mathrm{C}$ for $12 \mathrm{~h}$. The turbidity of Yellow River water as $6 \sim 7 \times 10^{3} \mathrm{CFU} / \mathrm{ML}$ and the bacterial assemblage of the Lanzhou section was mainly composed of fecal coliform ${ }^{18,19}$.

\section{Preparation of Co@Ag core-shell nanomaterials}

$70.8 \mathrm{mg}$ cobalt nanomaterials and $272 \mathrm{mg} \mathrm{AgNO}_{3}$ powder were accurately weighed and each dissolved in $50 \mathrm{~mL}$ deionized water. The two solutions were added to a three-pot flask, and the volume of reaction system was increased to $200 \mathrm{~mL}$.The mixture was stirred (800 rmp) for 4 h at room temperature. Co@Ag core-shell nanomaterials can be obtained after centrifugation at $5000 \mathrm{rmp}$. The samples were successively washed with deionized water and anhydrous ethanol by ultrasonic and centrifugation for 3 times, and then freeze-dried for further use.

\section{Characterization of Co@Ag core-shell nanomaterials}

The morphologies of the synthesized Co@Ag core-shell nanoparticle samples were characterized by transmission electron microscope (TEM, Kevex JSM-6701F, Japan). The suspended samples were dropped into carbon-coated copper grids, and the excess samples were removed from the grid by using a cone of a blotting paper. The prepared thin film was reserved in a grid box sequentially ${ }^{20}$. The type of elements, valence and mass ratio of Co@Ag core-shell nanomaterials were determined by X-ray photoelectron spectroscopy (XPS, ESCALAB 250, Thermo Fisher Scientific Technology, USA). The samples were compressed into a pellet of $2 \mathrm{~mm}$ thickness and then mounted on a sample holder by utilizing double-sided adhesive tape for XPS analysis. The sample holder was then placed into a fastentry air load-lock chamber without exposure to air and evacuated under vacuum overnight. Finally, the sample holder was transferred to the analysis chamber for XPS study ${ }^{21}$.The phase composition of the Co@Ag core-shell nanomaterials was determined by X-ray diffraction (XRD, Bruker D8 Advance). Dried powder of nanocomposites was drop-coated onto glass slide, and the XRD pattern was collected from $20^{\circ}$ to $80^{\circ}$ at $2 \theta$ angle. The machine voltage was $45 \mathrm{keV}$, the current was $20 \mathrm{~mA}$, and $\mathrm{Cu} \mathrm{Ka}(\lambda=$ $0.1542 \mathrm{~nm}$ ) radiation was used as an X-ray source ${ }^{20}$.The magnetic properties of Co@Ag core-shell nanomaterials were detected by an oscillating sample magnetometer at room temperature (VSM, Lakeshore Cryotronics Inc., Ohio, USA). The stability (Zeta potential) of Co@Ag core-shell nanomaterials was assessed using a dynamic light scattering (DLS) instrument (ZETASIZER Nanoseries Model ZEN 3600 , Malvern Instruments).

\section{Measurements of antibacterial properties of Co@Ag core-shell nanomaterials in vitro}

The standard strains of Pseudomonas aeruginosa, Escherichia coli, Staphylococcus aureus and Candida albicans were selected as representative bacteria for investigating the antibacterial properties of Co@Ag core-shell nanomaterials. The minimum inhibitory concentration (MIC) values of Co@Ag core-shell 
nanomaterials were determined by the disc agar diffusion method and broth dilution method ${ }^{21} .10 \mathrm{mg} / \mathrm{ml}$ of Co@Ag core-shell nanoparticle suspension were mixed into blank drug-sensitive paper, which was shook overnight to prepare drug-sensitive paper containing Co@Ag core-shell nanomaterials. The prepared drug-sensitive papers were pasted on the $\mathrm{M}-\mathrm{H}$ agar medium inoculated with the above four kinds of standard bacteria overnight to measure the antibacterial effect.

Under sterile conditions, a single colony of Pseudomonas aeruginosa, Escherichia coli, Staphylococcus aureus and Candida albicans were selected and diluted with LB liquid medium to reach a level of $\mathbb{Z}$ $1.0 \times 10^{6} \mathrm{CFU} / \mathrm{ML}^{22,23} .2 \mathrm{~mL}$ diluted bacterial solution was added to each well of the 24-well plate sequentially. Then, a certain amount of bacteriostatic solution containing Co@Ag core-shell nanomaterials was added in order and the concentrations of the bacteriostatic solution was as follows: 0 (positive control, bacteria solution added without Co@Ag core-shell nanomaterials), 100, 200, 300, 400, $500,600,700,900,1000,2000 \mathrm{~g} / \mathrm{ml}$, with the last well as a negative control (2ml Co@Ag core-shell nanomaterials bacteriostatic solution added); all the groups were with the duplicate holes set. The plates were incubated at $37^{\circ} \mathrm{C}$ on a rotary shaker $(180 \mathrm{rpm})$ for $12 \mathrm{~h}$. The antibacterial effect of Co@Ag coreshell nanomaterials was measured on the next day, and the minimum concentration without bacterial growth was the MIC.

The minimum inhibitory concentration (MIC) of Co@Ag core-shell nanomaterials for the Yellow River water: under sterile conditions, the Yellow River water was diluted into the LB liquid medium at 1:100. After that, $2 \mathrm{ml}$ diluted Yellow River water LB liquid medium was added to the 24-well plate, and then a certain amount of bacteriostatic solution containing Co@Ag core-shell nanomaterials was added sequentially. The bacteriostatic concentration of each well was as follows: 0 (positive control, only bacteria solution added without Co@Ag core-shell nanomaterials), 100, 200, 300, 400, 500, 600, 700, $900,1000,2000 \mathrm{~g} / \mathrm{ml}$, with the last well as negative control (2ml Co@Ag core-shell nanomaterials bacteriostatic solution added); all the groups were with the duplicate holes set. The plates were incubated at $37^{\circ} \mathrm{C}$ on a rotary shaker (180rpm) for $12 \mathrm{~h}$. The antibacterial effect of Co@Ag core-shell nanomaterials was measured on the next day, and the minimum concentration without bacterial growth was the MIC.

\section{Measurement of antibacterial properties of Co@Ag core-shell nanomaterials reclaimed}

Measurement of minimum inhibitory concentration (MIC) of Co@Ag core-shell nanomaterials reclaimed for Pseudomonas aeruginosa, Escherichia coli, Staphylococcus aureus and Candida albicans: After the MIC of Co@Ag core-shell nanomaterials were measured, the magnets were put under the 24-well plates for 30 min to make sure that Co@Ag core-shell nanomaterials were attracted at the bottom of the plate. After slowly discarding bacteria liquid in the 24 -well plates, $2 \mathrm{ml}$ of diluted bacteria liquid was added. The plates were incubated at $37^{\circ} \mathrm{C}$ on a rotary shaker (180 rpm) for $12 \mathrm{~h}$. The bacteriostatic effect of Co@Ag core-shell nanomaterials was observed by naked eyes on the third day, and the minimum concentration without bacteria growth was MIC. 
Measurement of minimum inhibitory concentration (MIC) of Co@Ag core-shell nanomaterials reclaimed for Yellow River water: After the MIC of Co@Ag core-shell nanomaterials for Yellow River water were measured, the magnets were put under the 24-well plates for 30 min to ensure that Co@Ag core-shell nanomaterials were attracted to the bottom of the plate. After slowly discarding the bacteria liquid in the 24-well plates, $2 \mathrm{ml}$ diluted bacteria liquid were added. The plates were incubated at $37^{\circ} \mathrm{C}$ on a rotary shaker (180 rpm) for 12h. The bacteriostatic effect of Co@Ag core-shell nanomaterials was observed by naked eyes on the third day, and the minimum concentration without bacteria growth was MIC.

\section{Measurements of the reclaim rate of reclaimed Co@Ag core-shell nanomaterials}

$5 \mathrm{mg}$ of Co@Ag core-shell nanomaterials were precisely weighed and dissolved into a centrifuge tube containing $25 \mathrm{~mL}$ Escherichia coli dilution solution with a bacterial concentration of $₫ 1.0 \times 10^{6} \mathrm{CFU} / \mathrm{ML}$. The bacteria were incubated at $37^{\circ} \mathrm{C}$ on a rotary shaker $(180 \mathrm{rpm})$ for $12 \mathrm{~h}$. The next day, the magnet was placed under the centrifuge tube for about 30min to make sure that Co@Ag core-shell nanomaterials were attracted to the bottom of the tube. Then slowly discarding the bacteria liquid in the centrifuge tube and dried the left Co@Ag core-shell nanomaterials at $60^{\circ} \mathrm{C}$ until the centrifuge tube was at constant weight. The reclaimed Co@Ag core-shell nanomaterials were weighed, and the reclaim rate was calculated according to the following formula: recovery $(\%)=$ recovery amount/input amount $\times 100 \%$.

\section{Results}

\section{Characterization of Co@Ag core-shell nanomaterials}

The morphology of Co@Ag core-shellnanomaterials characterized by TEM is shown in Figure 2-a. The outer surface of cobalt nanomaterials is covered with a layer of silver element in a "dendritic-like" formation. As shown in Figure 2-b, the composition of "dendritic" Co@Ag core-shell nanomaterials was relatively uniform in size, with an average particle size about $100 \mathrm{~nm}$.

The patterns of the X-ray diffraction (XRD) and X-ray photoelectron spectroscopy (XPS) of Co@Ag coreshell nanomaterials are shown in figure 3. Data displayed in Figure 3-a suggests that Co@Ag coreshell nanomaterials contain the characteristic peaks of both silver and cobalt elements, indicating that that the silver and cobalt elements contained in Co@Ag core-shell nanomaterials are successfully combined together. The average crystallite size of $\mathrm{Ag}$ and Co nanoparticle was 46 and $50.9 \mathrm{~nm}$, respectively, obtained by the Scherrer formula. Figure 3-b is the full spectrum of XPS, depending on analysis of the element distribution, the surface of Co@Ag core-shell nanomaterials contains three elements: $\mathrm{O}, \mathrm{Ag}$, and $\mathrm{Co}$, among which $\mathrm{Co}$ and $\mathrm{Ag}$ accounts for $9.04 \%$ and $10.03 \%$, respectively. The data was obtained by calculating the area under the $\mathrm{Co}$ and $\mathrm{Ag}$ wave peaks. The X-ray photoelectron spectrometer detected the superficial zone of the materials, and the surface of nanomaterials can be oxidized in the air, so 0 always resided in the Co@Ag core-shell nanomaterials ${ }^{24,25}$. Considering that, the patterns of XRD in Co@Ag core-shell nanomaterials indicate that only Ag and Co are the components of Co@Ag core-shell nanomaterials. The valence distribution of Ag contained in Co@Ag core- 
shell nanomaterials is shown in Figure 3-c. The valence of Ag exhibits $3 d_{5 / 2}$ and $3 d_{3 / 2}$, and its binding energy is $368.4 \mathrm{eV}$ and $374.4 \mathrm{eV}$, respectively. These levels of binding energy are consistent with their respective standard electron binding energies. The other positions whose binding energy are $374.43 \mathrm{eV}$ and $368.43 \mathrm{eV}$ correspond to the peak positions of $\mathrm{Ag}+$ in $\mathrm{Ag}_{2} \mathrm{O}^{24}$. The valence distribution of $\mathrm{CO}$ contained in Co@Ag core-shell nanomaterials is shown in Figure 3-d. Binding energies of 780.88eV and 796.9eV correspond to the $2 p_{3 / 2}$ and $2 p_{1 / 2}$ valence of $\mathrm{Co}$ in $\mathrm{Co}_{3} \mathrm{O}_{4}$, respectively, while binding energies of $786.58 \mathrm{eV}$ and $803.79 \mathrm{eV}$ are the satellite of $\mathrm{Co}$ in $\mathrm{Co}_{3} \mathrm{O}_{4}$, and binding energies $778.39 \mathrm{eV}$ and $793.49 \mathrm{eV}$ correspond to the peaks of the $\mathrm{Co}^{0}$ valence in elemental cobalt ${ }^{26}$.

The magnetic properties of Co@Ag core-shell nanomaterials are crucial for subsequent applications. Placing a magnet next to the container containing the Co@Ag core-shell nanomaterials suspension, result in the nanomaterials moving quickly to the side and close to the magnet under the magnetic force. This means that the Co@Ag core-shell nanomaterials have good magnetic responsiveness as shown in Figure 4-a. At room temperature, VSM was used to detect the magnetic properties of Co@Ag core-shell nanomaterials. The magnetic field range is $-30 \sim 30 \mathrm{kOe}$. We found that Co@Ag core-shell nanomaterials have magnetic responsiveness under an external magnetic field, and the magnetization saturation is $70.1 \mathrm{emu} / \mathrm{g}$ in Figure 4-c. No hysteresis was observed when the magnetic moment of the external magnetic field is 0 , which shows that the synthesized Co@Ag core-shell nanomaterials are superparamagnetic so that they can be separated from the solution by the external magnetic field. As shown in figure 4-b, Co@Ag core-shell nanomaterials have lower saturation magnetization than cobalt nanomaterials, which may be attributed to the fact that a layer of silver element attached to the surface of cobalt nanomaterials increases the thickness of the shell layer.

\section{Measurement of antibacterial effect of Co@Ag core-shell nanomaterials on standard strains and the Yellow River water}

The antibacterial effect of Co@Ag core-shell nanomaterials on common bacteria in daily life was evaluated through qualitative and quantitative methods. The results are shown in Figure 5 and Table 1: Co@Ag core-shell nanomaterials have good antibacterial effects on Staphylococcus aureus 3-a, Escherichia coli 3-b, Pseudomonas aeruginosa 3-c and Candida albicans 3-d; Co@Ag core-shell nanomaterials have significantly better antibacterial effects on Pseudomonas aeruginosa, Candida albicans and Staphylococcus aureus than Escherichia coli, and the diameter of the inhibition zone is $12.8 \pm 0.4,15.2 \pm 0.4,12.6 \pm 0.5,10.2 \pm 0.4 \mathrm{~mm}$, respectively.

From Table 1, we found that Co@Ag core-shell nanomaterials can achieve excellent antibacterial effects on general standard strains, and that the reclaimed Co@Ag core-shell nanomaterials could be reused still have good antibacterial effects on these bacteria. As well, Co@Ag core-shell nanomaterials can achieve excellent antibacterial effects on the Yellow River water, and the reclaimed Co@Ag core-shell nanomaterials still have antibacterial effects when reused.

Table $1 \mathrm{MIC}(\mu \mathrm{g} / \mathrm{mL})$ of Co@Ag Core-Shell nanomaterials for standard strains 


\begin{tabular}{|lll|}
\hline Co@Ag & MIC & \\
\cline { 2 - 3 } & First time to use & Reused \\
\hline Pseudomonas aeruginosa & 200 & 400 \\
\hline Staphylococcus aureus & 400 & 600 \\
\hline Escherichia coli & 500 & 700 \\
\hline Candida albicans & 200 & 400 \\
\hline Yellow River water & 700 & 900 \\
\hline
\end{tabular}

\section{Measurement of reclaim rate of Co@Ag core-shell nanomaterials}

Using a magnet after removing supernatant and drying 4.9mg out of the 5mg of Co@Ag core-shell nanomaterials originally added in the first antibacterial experiment resulted in a reclaim rate of $98 \%$.

\section{Discussion}

Nanobiotechnology is an emerging field; especially in the field of medicine. A large number of medicines get revived and applied widely due to the further study of nanomaterials, which have made great contributions to human life and health. Benefitting from the emergence of nanotechnology, common antibacterial materials are remoulded to be nanoscale antimicrobial materials which can be combined with antimicrobial carriers through certain methods and technologies, to be effective. Metal nanoantibacterial agents include many other heavy metal ions such as $\mathrm{Ag}^{+}, \mathrm{Zn}^{2+}, \mathrm{Cu}^{2+}, \mathrm{Hg}^{2+}$ and many other heavy metal ions, among which $\mathrm{Ag}+$ is the antibacterial agent showing the best antibacterial effect and the least toxicity to the human body. Hence the most widely studied antibacterial agents are those containing silver ions ${ }^{27,28}$. Recent studies have shown that silver ions have a strong killing effect on 12 kinds of gram-negative bacteria, 8 kinds of gram-positive bacteria and 6 kinds of molds. Due to its excellent antibacterial properties, silver has the potential to be an antibacterial material ${ }^{29}$. Magnetic nanomaterials have the advantages of uniform particle size, large surface area, superparamagnetism, and are widely used in cemented carbide, sewage treatment, batteries, permanent magnet materials, diamond tool manufacturing and other industries, as well as in the fields of catalysts, magnetic materials, absorbing materials, ceramics, and more ${ }^{30-32}$. Among them, compared with iron and nickel nanomaterials, cobalt nanomaterials have stronger magnetic properties and are easier to agglomerate and oxidize.

In this study, cobalt nanomaterials as the core Co@Ag core-shell nanomaterials can reduce silver ions in a $\mathrm{AgNO}_{3}$ solution to become elemental silver which can be coated on the surface of cobalt nanomaterials. The prepared Co@Ag core-shell nanomaterials have a uniform particle size of about $100 \mathrm{~nm}$, form as "dendritic" so that increasing the materials' surface area easily have contact with the outside world. In addition, the presence of silver on the surface of Co@Ag core-shell nanomaterials is 
higher, which ensures that the materials have excellent antibacterial effects on Pseudomonas aeruginosa, Escherichia coli, Staphylococcus aureus, Candida albicans and the bacteria in the water of the Yellow River. Moreover, Co@Ag core-shell nanomaterials have strong magnetic properties because of the existence of cobalt. Over the course of sewage treatment, Co@Ag core-shell nanomaterials can not only inhibit the growth of bacteria, but also can be reclaimed by the external magnetic field and the reclaim rate is up to $98 \%$, hence reducing the toxicity of metal silver. Surprisingly, the reclaimed Co@Ag core-shell nanomaterials still have a good antibacterial effect. In the long run, the reclaimed Co@Ag core-shell nanomaterials technology will greatly save enterprise production costs, and do not have side effects on the surroundings to reduce environmental pollution and disposal costs. The preparation method of Co@Ag core-shell nanomaterials is really simple, and the Co@Ag core-shell nanomaterials are easy to be recycled owing to their superparamagnetism. to decrease toxicity of left Ag such that the materials can be potential candidates for sewage treatment.

\section{Conclusion}

In conclusion, Co@Ag core-shell nanomaterials have both the antibacterial bioactivities of silver and the magnetic properties of cobalt to ensure that the nanomaterials can be recycled through the external magnetic field. Furthermore, the Co@Ag core-shell nanomaterials bring out significant antibacterial effects on the standard strain, with the growth of bacteria in water of the Yellow River also being inhibited. These also makes it possible to serve as a new type of recyclable antibacterial agent

\section{Declarations}

\section{Acknowledgments}

This work was supported by the National Natural Science Foundation of Shaanxi (No. 2019JQ-1000).

\section{Author contributions}

Y.Y.Z.: study design, data collection, analysis, and manuscript writing. Y.J.L., S.H., Q.G., Y.C.Z.: data collection and analysis. X.X.S.: manuscript review. G.C.Z.: study design, analysis, and manuscript review. All authors reviewed the manuscript.

\section{Competing interests}

The authors declare no conflict of interest.

\section{Consent for publication}

Not applicable.

\section{References}


1 Friedman, N. D., Temkin, E. \& Carmeli, Y. The negative impact of antibiotic resistance. Clin Microbiol Infect 22, 416-422, doi:10.1016/j.cmi.2015.12.002 (2016).

2 Jalvo, B., Faraldos, M., Bahamonde, A. \& Rosal, R. Antimicrobial and antibiofilm efficacy of selfcleaning surfaces functionalized by $\mathrm{TiO} 2$ photocatalytic nanoparticles against Staphylococcus aureus and Pseudomonas putida. J Hazard Mater 340, 160-170, doi:10.1016/j.jhazmat.2017.07.005 (2017).

3 Kaviyarasu, K. et al. Elucidation of photocatalysis, photoluminescence and antibacterial studies of ZnO thin films by spin coating method. J Photochem Photobiol B 173, 466-475, doi:10.1016/j.jphotobiol.2017.06.026 (2017).

4 Mousa, S. A. et al. Novel mycosynthesis of $\mathrm{Co304}, \mathrm{CuO}, \mathrm{Fe} 304$, NiO, and $\mathrm{ZnO}$ nanoparticles by the endophytic Aspergillus terreus and evaluation of their antioxidant and antimicrobial activities. Appl Microbiol Biotechnol 105, 741-753, doi:10.1007/s00253-020-11046-4 (2021).

5 Louwakul, P., Saelo, A. \& Khemaleelakul, S. Efficacy of calcium oxide and calcium hydroxide nanoparticles on the elimination of Enterococcus faecalis in human root dentin. Clin Oral Investig 21, 865-871, doi:10.1007/s00784-016-1836-x (2017).

6 Monzavi, A., Eshraghi, S., Hashemian, R. \& Momen-Heravi, F. In vitro and ex vivo antimicrobial efficacy of nano-MgO in the elimination of endodontic pathogens. Clin Oral Investig 19, 349-356, doi:10.1007/s00784-014-1253-y (2015).

7 Costa, E. M., Silva, S., Pina, C., Tavaria, F. K. \& Pintado, M. M. Evaluation and insights into chitosan antimicrobial activity against anaerobic oral pathogens. Anaerobe 18, 305-309 (2012).

8 Dubas, S. T., Wacharanad, S. \& Potiyaraj, P. Tunning of the antimicrobial activity of surgical sutures coated with silver nanoparticles. Colloids \& Surfaces A Physicochemical \& Engineering Aspects 380, 2528 (2011).

9 Ip, M., Lui, S. L., Poon, V. K. M., Lung, I. \& Burd, A. Antimicrobial activities of silver dressings: an in vitro comparison. J Med Microbio/ 55, 59-63, doi:10.1099/jmm.0.46124-0 (2006).

10 Sergevnin, V. I., Klyuchareva, N. M., Antipin, D. P. \& Laricheva, E. N. Comparative evaluation of the efficacy of uncoated and coated with silver silicone urethral catheters for prevention of urinary tract infections among patients of the intensive care unit. Urologiiஐa (Moscow, Russia: 1999), 33-36 (2016).

11 Poggio, C., Trovati, F., Ceci, M., Chiesa, M. \& Pietrocola, G. Biological and antibacterial properties of a new silver fiber post: In vitro evaluation. Journal of Clinical and Experimental Dentistry 9, e387-e393 (2017).

12 Wu et al. Fabrication of silver nanoparticles embedded into polyvinyl alcohol (Ag/PVA) composite nanofibrous films through electrospinning for antibacterial and surface-enhanced Raman scattering (SERS) activities. Materials Science \& Engineering C Materials for Biogical Applications (2016). 
13 Lansdown, A. A review of the use of silver in wound care: facts and fallacies. British Journal of Nursing 13, 6-19 (2004).

14 Zhang, X. G. et al. Constructing magnetic and high-efficiency Agl/CuFe2O4 photocatalysts for inactivation of Escherichia coli and Staphylococcus aureus under visible light: Inactivation performance and mechanism analysis. Sci Total Environ 668, 730-742, doi:10.1016/j.scitotenv.2019.03.068 (2019).

15 Atacan, K., Ozacar, M. \& Ozacar, M. Investigation of antibacterial properties of novel papain immobilized on tannic acid modified Ag/CuFe2O4 magnetic nanoparticles. Int J Biol Macromo/ 109, 720 731, doi:10.1016/j.ijbiomac.2017.12.066 (2018).

16 Wang, T. et al. Facile loading of Ag nanoparticles onto magnetic microsphere by the aid of a tannic acid-Metal polymer layer to synthesize magnetic disinfectant with high antibacterial activity. $J$ Hazard Mater 342, 392-400, doi:10.1016/j.jhazmat.2017.08.047 (2018).

17 Cha, S., Mo, C., Kim, K. \& Hong, S. Ferromagnetic cobalt nanodots, nanorices, nanowires and nanoflowers by polyol process. Journal of Materials Research 20, 2148-2153 (2005).

18 Wang LR, Liu FM \& Zhang J. Study on the Present Condition of the Fecal Coliform Pollution in the Lanzhou Section of the Yellow River. Environmental Science \& Technology v.33, 63-67.

19 Jia, R. L. Water Quality Analysis and Pollution Prevention Measures in Lanzhou Section of Yellow River. Journal of Anhui Agricultural Sciences (2010).

20 AbdelmoneimBakur, TarigElshaarani, YongwuNiu \& QiheChen. Comparative study of antidiabetic, bactericidal, and antitumor activities of MEL@AgNPs, MEL@ZnONPs, and Ag-ZnO/MEL/GA nanocomposites prepared by using MEL and gum arabic. RSC Advances 9 .

21 Rastogi, S. K. et al. Ag colloids and Ag clusters over EDAPTMS-coated silica nanoparticles: synthesis, characterization, and antibacterial activity against Escherichia coli. Nanomedicine Nanotechnology Biology \& Medicine 7, 305-314 (2011).

22 Syed, F. S., Kasabe, A. M., Mane, P. C., Chaudhari, R. \& Adhyapak, P. V. Selective antifungal and antibacterial activities of $\mathrm{Ag}-\mathrm{Cu}$ and $\mathrm{Cu}-\mathrm{Ag}$ core-shell nanostructures synthesized in-situ PVA. Nanotechnology 31 (2020).

23 Gong, P. et al. Preparation and antibacterial activity of Fe304 and Ag nanoparticles. (2007).

24 Zhao, X., Wu, P., Lei, Y., Chen, F. \& Liu, Y. Sun-Light-Driven Plasmonic Ag/AgCl@TNT Photocatalysts for High-Efficient Absorption-Regeneration and Photocatalytic Degradation. Applied Surface Science 529, 147010 (2020).

25 Zhou, X. et al. Co-effects of $\mathrm{C} / \mathrm{Ag}$ dual ion implantation on enhancing antibacterial ability and biocompatibility of silicone rubber. Biomed Mater 15, 065003, doi:10.1088/1748-605X/ab99d3 (2020). 
26 Zhang, Y. et al. Carbon nanofibers supported Co/Ag bimetallic nanoparticles for heterogeneous activation of peroxymonosulfate and efficient oxidation of amoxicillin. J Hazard Mater 400, 123290, doi:10.1016/j.jhazmat.2020.123290 (2020).

$27 \mathrm{Li}, \mathrm{W}$. R. et al. Antibacterial activity and mechanism of silver nanoparticles on Escherichia coli. App/ Microbiol Biotechnol 85, 1115-1122, doi:10.1007/s00253-009-2159-5 (2010).

28 Bosetti, M., Masse, A., Tobin, E. \& Cannas, M. Silver coated materials for external fixation devices: in vitro biocompatibility and genotoxicity. Biomaterials 23, 887-892, doi:10.1016/s0142-9612(01)00198-3 (2002).

29 You, C. et al. The progress of silver nanoparticles in the antibacterial mechanism, clinical application and cytotoxicity. Mol Biol Rep 39, 9193-9201, doi:10.1007/s11033-012-1792-8 (2012).

30 Xiao, J., Chernova, N. A. \& Whittingham, M. S. Layered Mixed Transition Metal Oxide Cathodes with Reduced Cobalt Content for Lithium lon Batteries. Chemistry of Materials 20, 7454-7464 (2008).

31 Harris, V. G. et al. High coercivity cobalt carbide nanoparticles processed via polyol reaction: A new permanent magnet material. Physics volume 43, 165003-165009(165007) (2010).

32 Imine, S. et al. Bottom-up and new compaction processes: A way to tunable properties of nanostructured cobalt ferrite ceramics. Journal of the European Ceramic Society 31, 2943-2955 (2011).

\section{Figures}


a

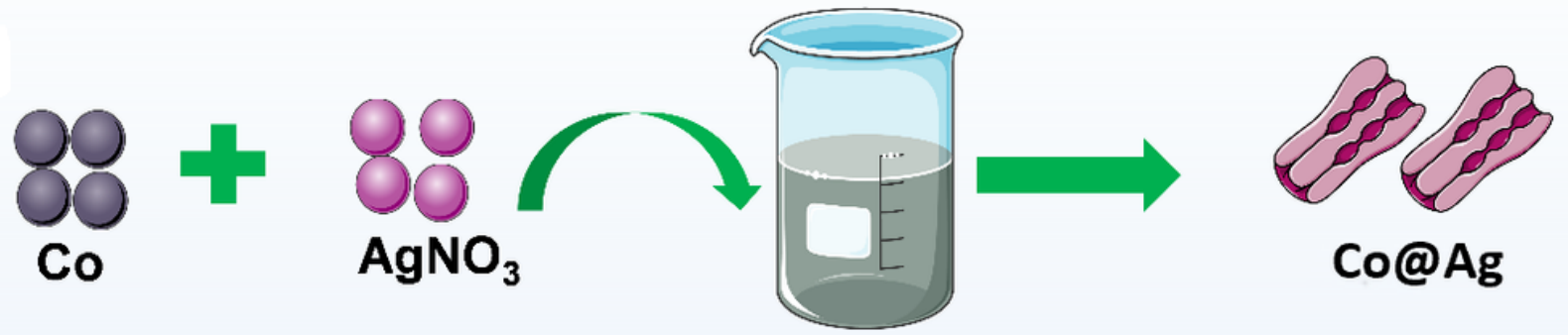

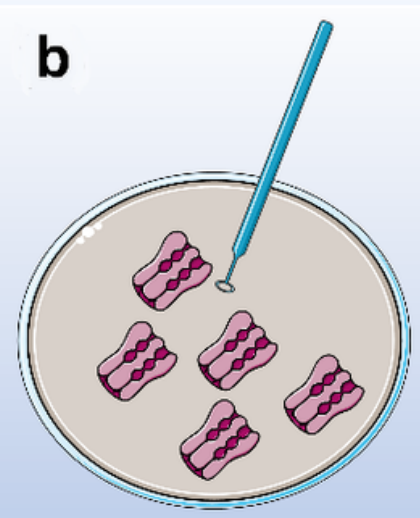

Petri dish

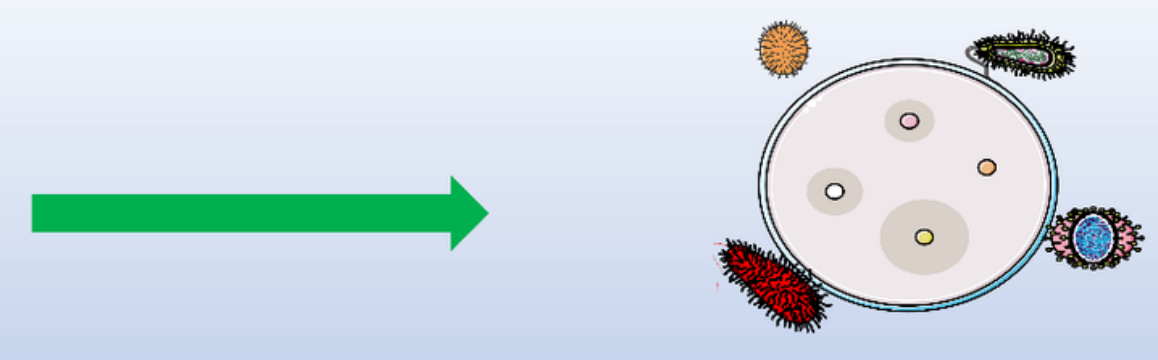

\section{Antibacterial}

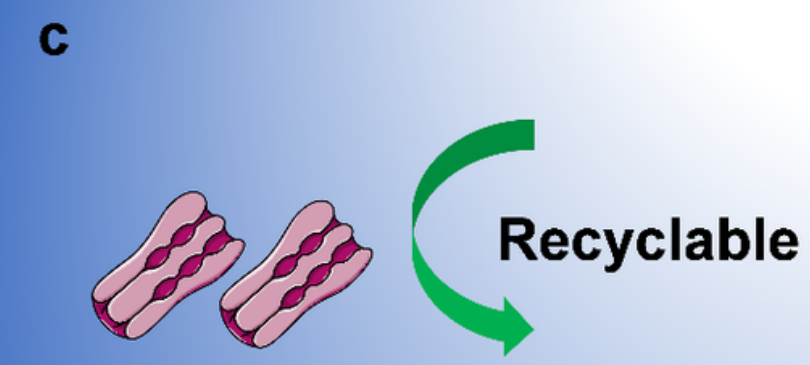

Co@Ag

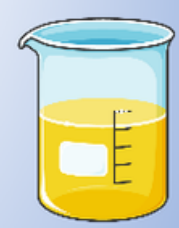

自

Yellow River Water Purification

Figure 1

Fabrication process and schematic of the Co@Ag core-shell nanomaterials 

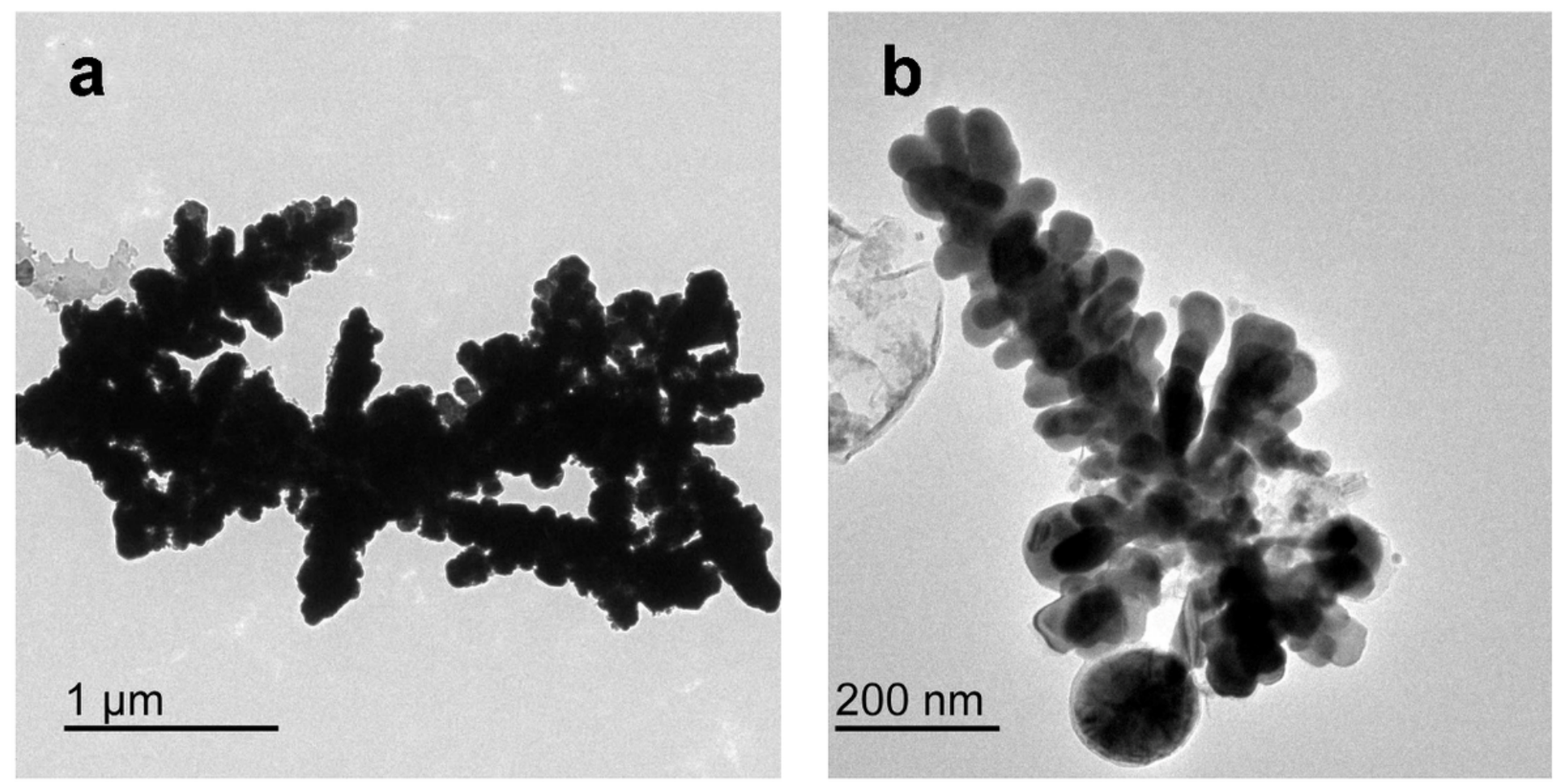

Figure 2

TEM images of Co@Ag core-shell nanomaterials 

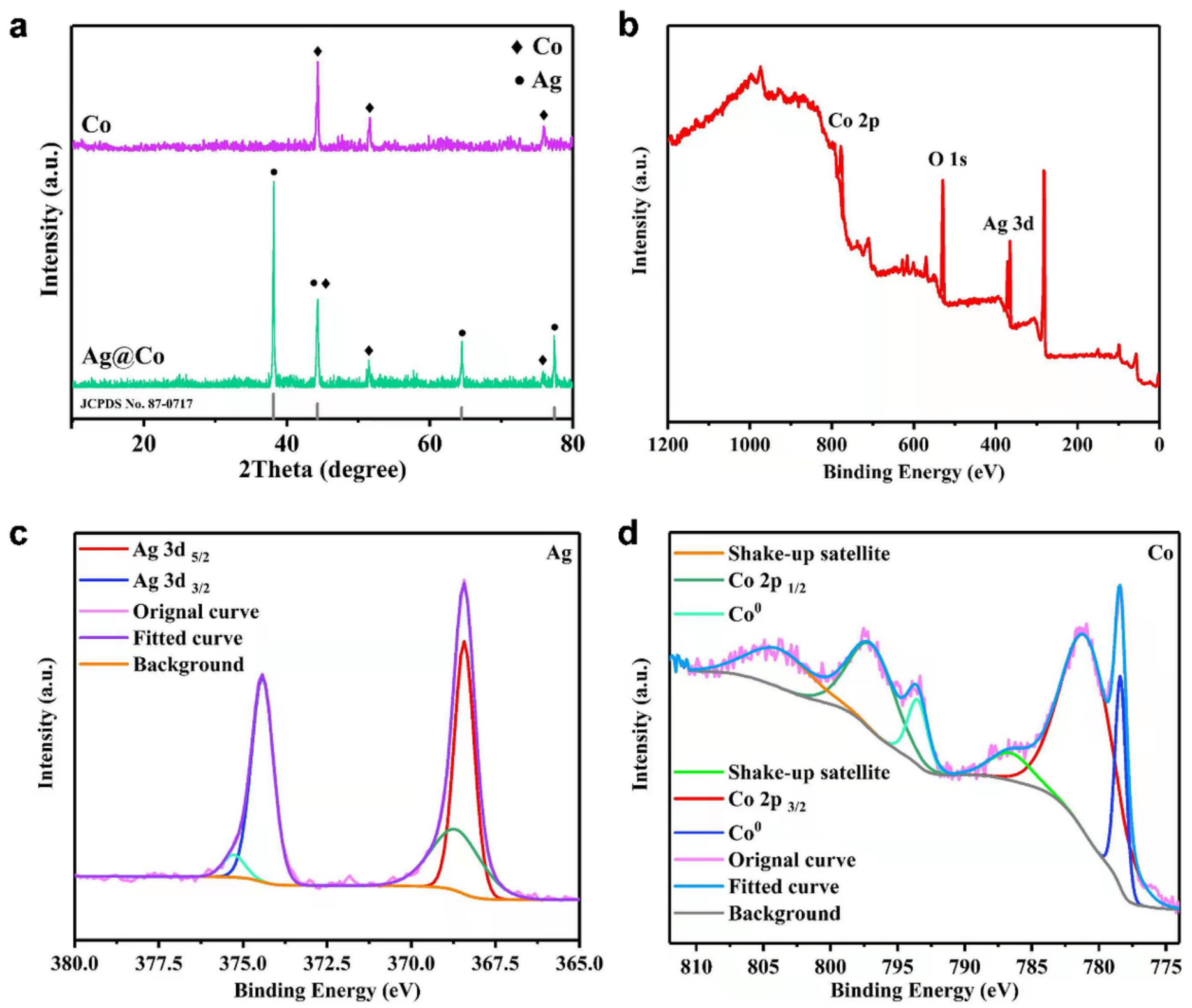

Figure 3

XRD/XPS spectra of Co@Ag Core-Shell nanomaterials 


\section{a}

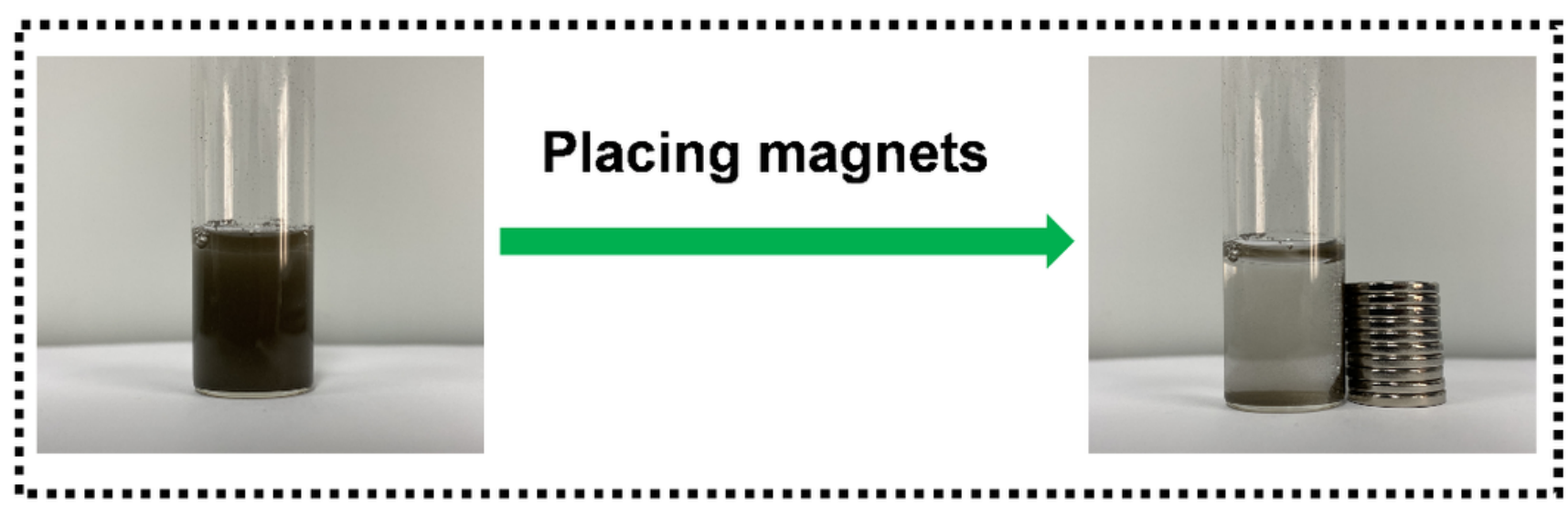

b

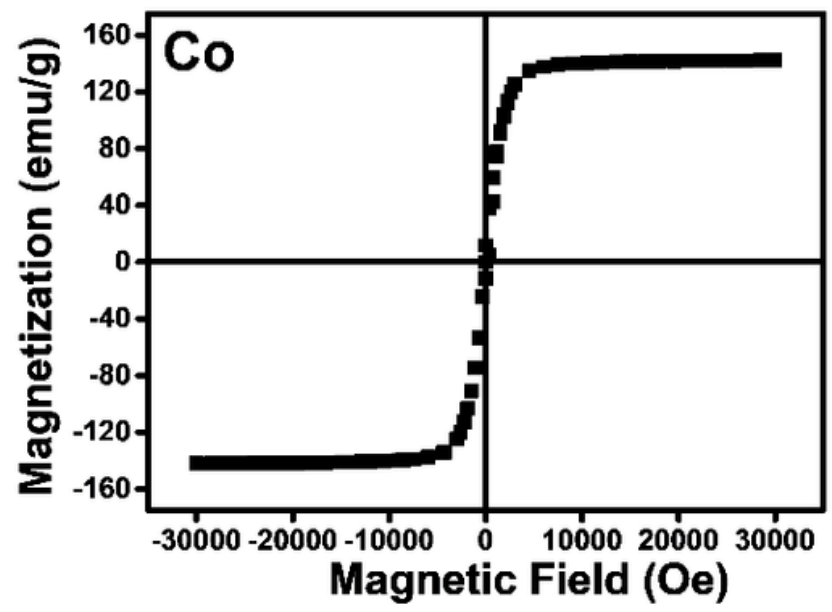

C

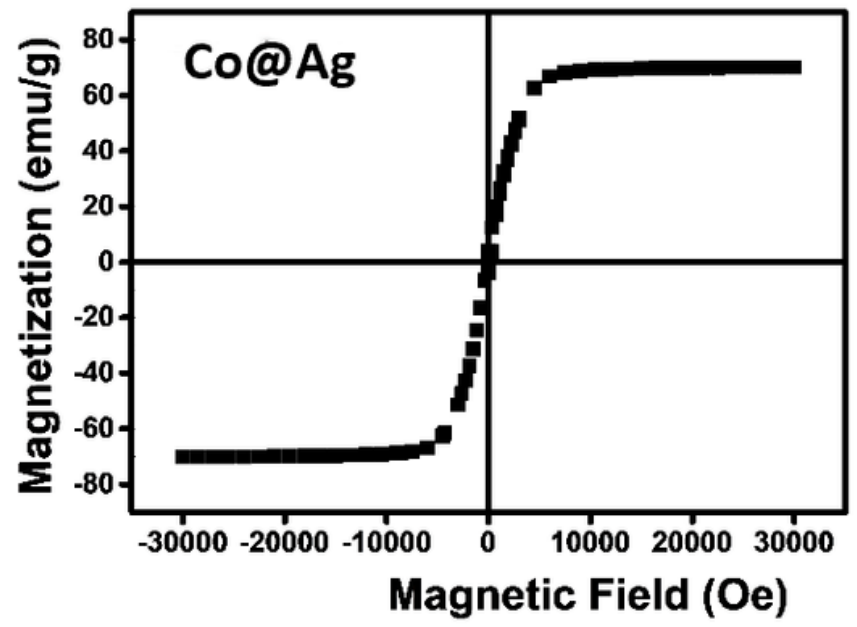

Figure 4

Co@Ag core-shell nanomaterials Schematic diagram of magnetic properties of nanomaterials
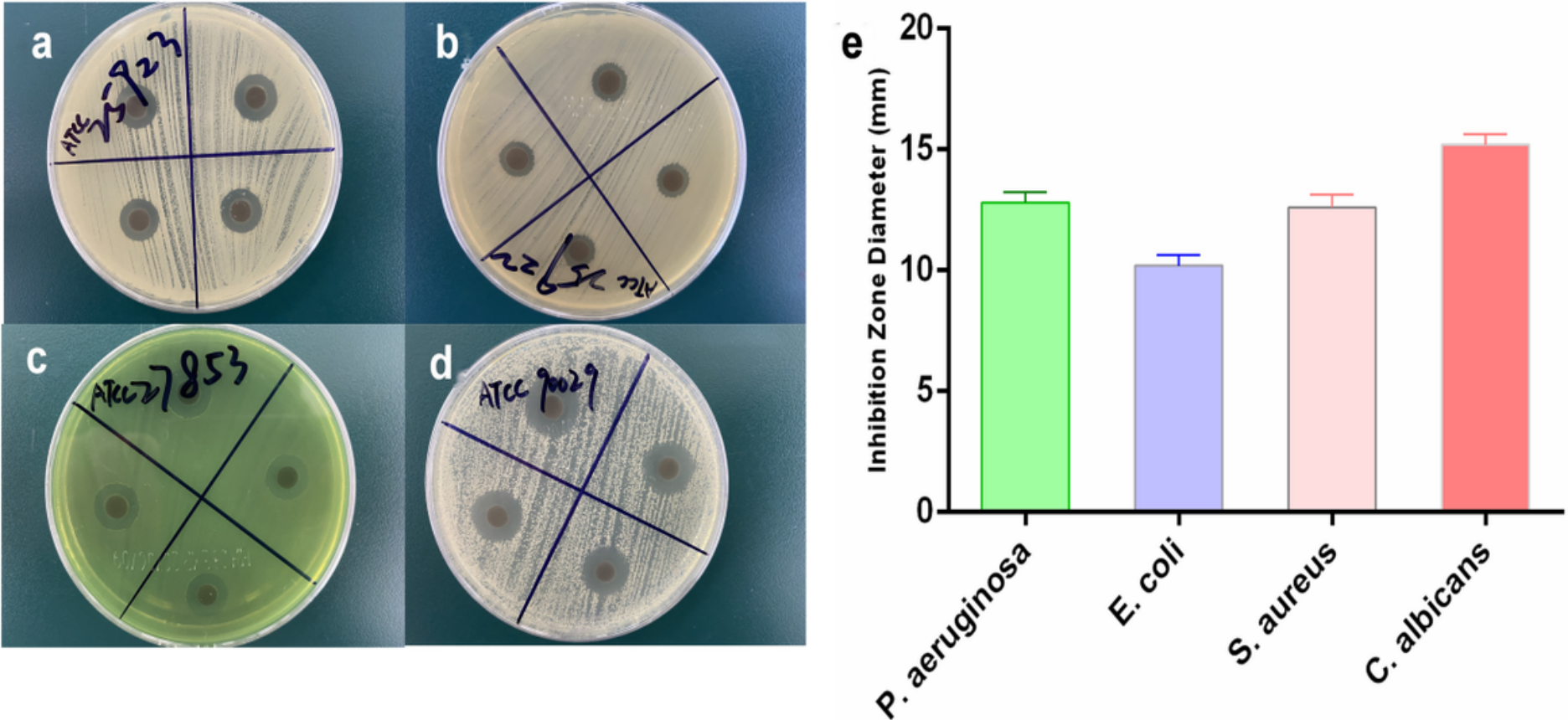
Figure 5

Schematic diagram of antibacterial properties of Co@Ag Core-Shell nanomaterials on standard strains 